\title{
SURFACE INSTABILITIES IN FINITELY STRAINED SOLIDS UNDER STATIC LOADING
}

\author{
NICOLAS TRIANTAFYLLIDIS \\ Department of Aerospace Engineering, The University of Michigan
}

ABSTRACT Surface bifurcation in a quasistatically loaded solid is an important instability phenomenon occurring at high levels of strain. After a discussion of theoretical as well as experimental work done in this area, a general formulation for the surface buckling of an incrementally linear three dimensional halfspace is presented and corresponding necessary conditions for the onset of this instability are derived.

\section{Introduction and Motivation}

A very interesting stability phenomenon occurring during quasistatic straining of solids at adequately high strain levels, is the development of a surface waviness on their traction free boundaries. The importance of this phenomenon--which hereby will be referred to as surface instability or surface buckling--lies in the fact that it precedes, and subsequently,triggers more severe kinds of instability (e.g., shear localization) which ultimately lead to the failure of the solid in question.

In the first part of this paper, a somewhat lengthy review of the work done thus far on the subject of surface buckling will be presented in an effort to show the importance of this pheonomenon as a triggering mechanism for the failure of a large class of solids. In the second part, a general mathematical analysis of the surface buckling problen for any three dimensional incrementally linear solid is given and necessary conditions for the onset of this instability are derived. To the best of our knowledge, only sufficient conditions for the onset of surface buckling have been presented in the literature so far and that for the case where the solid enjoys certain symetries. An interesting consequence of our general analysis is the theoretical explanation of the experimentally observed fact that the resulting surface wrinkles are in the form of rather long parallel waves with a very short wavelength.

\section{Analytical and Experimental Investigations Involving Surface Buckling in Solids}

The possibility of a plane, traction free surface of a homogeneously strained solid to lose flatness and develop surface wrinkles (i.e., waves) at adequately high levels of strain was first noted by Biot [1] in his study of the plane strain deformation of hyperelastic solids. Biot's analysis of the traction free infinite halfspace problem shows that, as the parallel to the free surface stress increases (in absolute value that is), it reaches a level where bifurcation becomes possible in the form of a sinusoidal surface wave of arbitrarily short or long wavelength. The corresponding eigenmodes decay exponentially beneath the surface with a wavelength of the same order as the one in the surface variation.

In a subsequent investigation of bifurcation phenomena in the plane strain tension test, Hill and Hutchinson [2] found the following interesting behavior which is common to a wide class of incrementally linear solids (hyperelastic as well as rate independent elasticplastic solids fall into this category). In the course of deformation of an orthotropic plane rectangular block with two opposite sides traction free and the other two sides shear 
traction free, while constrained to remain straight, the below described sequence of events takes place as the two constrained ends are being pulled apart. The first bifurcated solution occurs after the tensile load reaches $i$ ts maximum value and is in the form of diffuse necking. Subsequently, different bifurcation (with respect to the uniform strain configuration) eigenmodes become available as the distance separating the two end faces of the rectangular solid increases and, finally, with the material still in the elliptic regime* a surface bifurcation at the free ends becomes possible. Upon continuation of the deformation, the material loses ellipticity and, thus, failure by shear localization becomes possible. An exactly analogous type of behavior for the compression case has been found by Young [3]. The possibility for surface buckling in a finitely strained rectangular isotropic incompressible hyperleastic block was also found by Sawyers [4].

In a subsequent numerical (using the f.e.m.) investigation, Tvergaard, Needleman and Lo [5] have established the connection between the surface buckling phenomenon and the onset of failure by shear localization in the case of a hyperelastic as well as a rate independent elastic-plastic rectangular block under plane strain tension. By assuming a slightly imperfect block, i.e., a block whose free surface has a sinusoidal undulation of negligibly small (compared with the block's dimensions) amplitude, they found that as the strain in the block increased near the critical level for the onset of a surface bifurcation, the strain pattern started becoming increasingly non-uniform with the surface wave amplitudes growing rapidly. Eventually, the strain near the region of one of the wavelets became the maximal one for the entire body and soon reached the critical level for shear localization, at which point a shear band with size comparable to that of the surface wavelength started propagating inwards.

Surface buckling plays a very important role in the stability analysis of a plate subjected to finite bending. In a theoretical investigation of the bifurcation phenomena associated with the finite strain pure bending test of a thick plate (obeying constitutive laws of the type considered in [2]) under plane strain conditions, Triantafyllidis [6] found that, as the plate's curvature increases, the first bifurcation encountered is a surface bifurcation on the compressive zone of the plate which is followed by (as the curvature keeps increasing) a similar instability on the tensile zone. Upon further increase of the curvature, the outer zones of the plate lose their ellipticity. In a subsequent numerical investigation of this phenomenon using finite elements, Triantafyllidis, Needleman and Tvergaard [7] showed (using a slightly imperfect plate as in [5]) that as the curvature increases past the critical ones for the onset of the surface buckling at the two surfaces of the plate, the amplitude of the surface ondulations grows very rapidly. strain at one of the surface ripples dominates and eventually reaches the critical level for loss of ellipticity in the material. At higher curvatures, the deformation pattern is strongly reminiscent of a plastic hinge type of failure.

A number of investigations where the onset of a surface instability plays an important role in the body's failure mechanism have followed, much in the spirit of the initial work in [2] and [5]. We mention here the work by Larsson, Needleman, Tvergaard and Storakers [8] on the instability of internally pressurized ductile thick metal cylinders where a surface bifurcation on the inner surface of the tube provided enough strain localization in order to trigger a shear band type of failure.

Quite recently, Tvergaard [9] using a more sophisticated rate independent plasticity model (than in the above studies) capable of incorporating void nucleation and growth

*By abuse of language we say that a material is elliptic at a given stage of deformation if the corresponding incremental equilibrium equations are elliptic. 
analyzed the ductile shear fracture at a free surface. Again in this case, the strain localization mechanism which triggers shear bands is a surface bifurcation.

In all the aforementioned works, the boundary value problems considered involved the plane strain approximation. Hutchinson and Tvergaard [10] have investigated surface bifurcation in an infinite incompressible halfspace using two different finite strain versions of the $I_{2}$ deformation theory of plasticity. In their investigation, where the material considered was always orthotropic with one axis of orthotropy perpendicular to the free surface, they examined the possibility of bifurcation only for some predetermined type of eigenmode. A similar type of analysis involving a surface bifurcation in a non-plane strain problem was presented by Bassani, Durban and Hutchinson [11] for the instability of a pressurized cavity. There, as in $[6]$, the surface instability was the first one encountered as the cavity volume increases.

Experimental observations involving surface irregularities that develop at adequately high strain levels in statically loaded solids have repeatedly been reported in the past.

Hahn and Rosenfield [12] have observed strain induced surface irregularities in plane strain tension of ductile metals. These wavelike irregularities, which they call superbands, run perpendicular to the maximum tensile direction and are about one grain wide and many grains long. Microcracks were observed, which, upon continuation of the loading, developed into a shear crack type failure much the same way as in the calculations reported in [5].

As far as observations of surface instabilities in the case of finite bending of metallic bars, wrinkles on the compressed zone of the bar have been observed by Mylonas [13] (see also [14]). More recent experiments on finite bending of aluminum bars by Hutchinson and Tvergaard [10] also produced surface buckling on the tensile site of the bars.

A very nice pattern of surface wrinkles appears in some of the specimens of Larsson [15] during an experimental investigation of stability in thick aluminum and copper tubes subjected to internal pressure. In these experiments the axisymmetric state of deformation bifurcates into an eccentric shape and during the final collapse of the tube, considerable bulging in one side is observed until fracture occurs. In that highly strained bulged region, surface waves parallel to the tube axis have been observed in the case of the aluminum specimens (but not for the copper ones) prior to fracture.

\section{Mathematical Analysis of the Surface Bifurcation}

Here the general problem for the surface bifurcation of a homogeneous, incrementally linear three dimensional halfspace will be formulated and necessary conditions for the onset of this instability will be derived.

Consider an infinite body whose current configuration occupies the halfspace $x_{3} \leq 0$. The bifurcation eigenmode $u_{i}\left(x_{1}, x_{2}, x_{3}\right)$ for the traction free halfspace problem has to satisfy the following incremental equilibrium equations inside the halfspace (e.g., see [10])

$$
L_{i j k \ell} u_{k, \ell j}=0 * \quad\left(x_{\alpha} \in \mathbb{R}, x_{3} \leq 0\right)
$$

while the boundary conditions on the traction free surface are

$$
\mathrm{L}_{i 3 k \ell} \mathrm{u}_{k, \ell}=0 \quad\left(\mathrm{x}_{\alpha} \in \mathbb{R}, \mathrm{x}_{3}=0\right)
$$

\footnotetext{
*Note: Here and subsequently the usual convention of employing Greek indices, if their range is from 1 to 2 and Latin ones if their range is from 1 to 3, is adopted. Summation of repeated indices is tacitly assumed unless an index is enclosed between parentheses. A comma followed by an index denotes partial differentiation with respect to the corresponding coordinate.
} 
In aidition, for $u_{i}$ to be a surface mode, it has to satisfy

$$
u_{i} \rightarrow 0, \quad u_{i, j} \rightarrow 0 \quad \text { as } \quad x_{3} \rightarrow-\infty
$$

The (constant) incremental moduli $L_{i j k \ell}$ may in general depend on material properties, current stresses and the deformation history of the material up to the present state, in the case of a rate independent elastic-plastic material. In accordance with standard procedures in the theory of bifurcation, a monotonically increasing scalar load parameter $\lambda$ also termed as the "time like" parameter will be assumed to uniquely characterize, for a given deformation history, the present state of the material. In the initial stress-free state of the material, $\lambda$ is taken to be zero and subsequently increases monotonically as the loading process evolves Thus, the spatially constant incremental moduli will be considered to be functions of the load parameter, i.e., $L_{i j k \ell}(\lambda)$.

Assuming that the buckling eigenmode $u_{i}$ is twice continuously differentiable and uniformly bounded on the halfspace $x_{3} \leq 0$, one can ensure the existence of the Fourier transform $\hat{u}_{i}\left(w_{\alpha}, x_{3}\right)=\mathcal{F}\left(u_{i}\left(x_{\alpha}, x_{3}\right) ; x_{\alpha} \rightarrow \omega_{\alpha}\right\}$ in the sense of distributions (see Schwartz [16]). Although $u_{i}$ is a function in the classical sense, $\hat{u}_{i}$ will not generally be so and, thus, the subsequent equations are to be understood in the distribution sense. On taking the Fourier transform of the incremental equilibrium equations (3.1), one obtains the following system of ondinary differential equations with respect to $x_{3}\left(x_{3} \leq 0\right)$

$$
\left(-i \omega_{\alpha}\right)\left(-i \omega_{B}\right) L_{i \alpha k \beta} \hat{u}_{3}+\left(-i \omega_{\gamma}\right)\left(L_{i 3 k \gamma}+L_{i \gamma k 3}\right) \hat{u}_{k, 3}+L_{i 3 k 3} \hat{u}_{k, 33}=0
$$

The general solution to the above system of equations when (3.3) is also taken into account turns out to be

$$
\hat{u}_{k}\left(\omega_{\alpha}, x_{3}\right)=\sum_{j=1}^{3} \xi_{(j)} A_{k}^{(j)} \exp \left(-i c z_{(j)} x_{3}\right)
$$

where $z_{(j)}$ are the three roots with positive real parts of the following sixth order algebraic equation:

$$
\begin{aligned}
& \operatorname{Det}\left[L_{i \alpha k \beta} \stackrel{\star}{\omega}_{\alpha}{\stackrel{*}{\omega_{\beta}}}_{\beta}+\left(L_{i 3 k \gamma}+L_{i \gamma k 3}\right) \stackrel{*}{\omega}_{\gamma} z+L_{i 3 k 3} z^{2}\right]=0 \\
& \text { with } \stackrel{\star}{\omega}_{1} \equiv \cos \Omega, \stackrel{\star}{\omega}_{2} \equiv \sin \Omega \text { and } \omega_{\alpha}=c \stackrel{\star}{\omega}_{\alpha} \quad \text { where } \mathrm{c}>0
\end{aligned}
$$

The requirement that all the roots of (3.6) should be nonreal stems from the fact that we are interested only on surface bifurcations in the elliptic regime of the material and the condition that (3.6) admits no real root ensures the ellipticity of the incremental equilibrium equations (3.1). Thus the $z_{(j)}$ in (3.5) are of the form

$$
z_{(j)}=\alpha_{j}(\Omega)+i B_{j}(\Omega) ; B_{j}>0
$$

The (complex) constant vectors $A_{k}^{(j)}(\Omega)$ can, without loss of generality, be chosen to have unit norm and should also satisfy the system

$$
\left[L_{i \alpha k \beta} \stackrel{\star}{\omega}_{\alpha} \stackrel{\star}{\omega}_{B}+\left(L_{i 3 k \gamma}+L_{i \gamma k 3}\right) \stackrel{\star}{\omega}_{\gamma} z_{(j)}+L_{i 3 k 3} z_{(j)}^{2}\right] A_{k}^{(j)}=0
$$

while the $\xi_{(j)}$ entering $(3.5)$ are unknown distributions* depending on $\omega_{\alpha}$ or equivalently on $c, \Omega, i . e ., \xi_{(j)}=\xi_{(j)}\left(\omega_{\alpha}\right)$.

\footnotetext{
*All distributions involved in this argument are temperate ones in view of the boundedness of $u_{i}$. An outline of the proof leading to the necessary conditions for surface bifurcation is given here with no attempt of further mathematical rigorization, which although possible, lies outside our scope.
} 
The Fourier transform of the boundary condition (3.2) yields

$$
\left(-i \omega_{\alpha}\right) L_{i 3 k \alpha} \hat{u}_{k}+L_{i 3 k 3} \hat{u}_{k, 3}=0 \quad\left(x_{3}=0\right)
$$

which combined with (3.5) provides the following necessary condition for the existence of a surface bifurcation:

$$
\sum_{j=1}^{3} s_{i j}(\Omega) \xi_{(j)}\left(\omega_{\alpha}\right)=0 ; s_{i j}(\Omega) \equiv\left[L_{i 3 k \alpha} \stackrel{*}{\omega}_{\alpha}+L_{i 3 k 3} z_{(j)}\right] A_{k}^{(j)}
$$

Equation (3.10) has to hold in the $\omega_{1}, \omega_{2}$ plane. The only possible way that this can be satisfied is if $\xi_{(j)}$ has a line support, $i . e$.

$$
\operatorname{supp}\left(\xi_{(j)}\right)=\left\{\omega_{1}= \pm c \cos \Omega_{0}, \omega_{2}= \pm c \sin \Omega_{0}\right\} ; c>0, \Omega_{0} \in(0, \pi)
$$

with the angle $\Omega_{0} \in[0, \pi)$ satisfying

$$
\operatorname{Det}\left[s_{i j}\left(\Omega_{0}\right)\right]=0
$$

Thus as it follows from our construction of the Fourier transform of the solution to the surface bifurcation problem, the existence of a real angle $\Omega_{0} \epsilon(0, \pi)$ is a necessary condition for the existence of a bifurcation eigenmode satisfying (3.1) - (3.3). Thus in the case of the traction free halfspace with an incrementally linear constitutive behavior, a surface bifurcation will first become possible for the minimum load parameter $\lambda$ for which a solution to (3.12) exists, i.e., the critical load $\lambda_{\text {cr }}$ will satisfy:

$$
\begin{gathered}
\text { Det }\left[s_{i j}(\lambda, \Omega)\right] \neq 0 ; \Omega \in[0, \pi), 0 \leq \lambda \leq \lambda_{c r} \\
\quad \operatorname{Det}\left[s_{i j}\left(\lambda_{c r}, \Omega_{0}^{c r}\right)\right]=0 ; \Omega_{0}^{c r} \in[0, \pi)
\end{gathered}
$$

The above procedure for the specification of the critical load corresponding to the onset of the surface bifurcation is applicable to any incrementally linear material without appealing to any symmetry property of the solid in contrast with the already existing analyses for the problem (see for example [10]). This approach is particularly advantageous in the case of general anisotropic materials as one can see in [17] where a plane strain version of this method has been employed.

Finally, an interesting remark is made about the shape of the corresponding eigenmode at the onset of the surface bifurcation. Assuming for simplicity that $\Omega_{0}^{c r}$ in (3.13) is unique and using the fact that $\xi_{(j)}\left(\omega_{\alpha}\right)$ has a line support (on $\left.-\omega_{1} \sin n_{0}^{c r}+\omega_{2} \cos n_{0}^{c r}=0\right)$ as well as the fact of the uniform boundedness for $u_{k}$, one can deduce that (see Schwartz [16]) $\xi_{(j)}$ can be put in the form

$$
\xi_{(j)}\left(\omega_{\alpha}\right)=\xi_{(j)}^{t}\left(\omega_{1} \cos \Omega_{0}^{c r}+\omega_{2} \sin \Omega_{0}^{c r}\right) \delta\left(-\omega_{1} \sin \Omega_{0}^{c r}+\omega_{2} \cos \Omega_{0}^{c r}\right)
$$

where 6 is Dirac's distribution. Employing (3.14) into (3.5) and upon inversion of the Fourier transform, one will obtain that

$$
u_{k}=u_{k}\left(x_{1} \cos \Omega_{0}^{c r}+x_{2} \sin \Omega_{0}^{c r}, x_{3}\right)
$$

which indicates that on the halfspace's surface, the bifurcation eigenmode is in the form of waves running parallel to the $-x_{1} \sin n_{0}^{c r}+x_{2} \cos n_{0}^{c r}$ direction. The amplitude of these waves, as well as their shape, is not determined by this analysis. In the experimental observations mentioned before the width of the parallel surface ripples is several gain sizes (in the case of metal polycrystals) while their length is orders of magnitude longer exactly 
as indicated by (3.15). (In the case of a nonunique $\Omega_{0}^{\mathrm{cr}}$, more than one such set of parallel surface waves will exist.)

\section{Conclusions}

Surface bifurcations are an important physical mechanism that precedes and subsequently triggers through strain localization, more severe types of instability (such as shear bands, fractured zones, etc.) when the maximum strain occurs on the solid's free surface. The necessary conditions for the onset of this phenomenonin any three dimensional incrementally linear solid have been derived, and a theoretical explanation for the observed shape of the bifurcation eigenmode has been established. The generality of the proposed method makes it particularly useful for the study of surface bifurcations in generally anisotropic solids where the methods that are available in the literature thus far fail to apply, in view of the required material symmetry and restrictions on the form of the assumed eigenmodes.

\section{$\underline{\text { References }}$}

[1] BIOT, M.A., Mechanics of Incremental Deformation, New York, Wiley, 1965.

[2] HILL, R. and HUTCHINSON, J.W., J: Mech. Physics Solids, 23, p. 234, 1975.

[3] YOUNG, N.J.B., Ibid, 24, p. 77, 1976.

[4] SAWYERS, K.N., Finite Elasticity, AMD Vol. 27, (R.S. Rivlin, Ed.,), p. 103, ASME, NY, 1977.

[5] TVERGAaRd, V., NEEDleman, A. and LO, K.K., J. Mech. Physics Solids, 29, p. 115, 1981.

[6] TRIANTAFyllidis, N., J. Mech. Physics Solids, 28, p. 221, 1980.

[7] TRIANTAFYLLIDIS, N., NEEDLEMAN, A. and TVERGAARD, V., Int'l. J. Solids and Structures, 18, p. 121,1982 .

[8] LARSSON, M., NEEdleman, A., TVERGAard, V. and STORAKERS, B, J. Mech. Physics Solids, 30 , p. 121, 1982.

[9] TVERGAARD, V., Ibid, 30, p. $399,1982$.

[10] HUTChINSON, J.W. and TVERGAARD, Int'1. J. Mech. Sci., 22, p. 339, 1980.

[11] BASSANI, J.J., DURBAN, D. and huTCHINSON, J.w., Math. Proc. Cambridge Phil. Soc., 87, p. 339,1980 .

[12] HAHN, G.T. and ROSENFIELD, A.R., Met. Trans. A 6A, p. 653, 1975.

[13] MYLONAS, C., Private communication, refers to work with Ludley and Drucker.

[14] HAWLEY, R.H. and DRUCKER, D.C., Experimental Mechanics, 13, p. 1, 1973.

[15] LARSSON, M., Ph.D. Thesis, Dept. of Strength of Matls. and Solid Mech., The Royal Institute of Technology, Stockhold, 1978 (in Swedish).

[16] SCHWARTZ, L. Theorie Des Distributions, Paris, Herrman, 1950.

[17] TRIAntafyllidis, N. and AbeyaratNe, R., J. Appl. Mech., 50, p. 149, 1983. 